\title{
SUBLINEAR FUNCTIONALS ERGODICITY AND FINITE INVARIANT MEASURES
}

\section{G. DAS}

\author{
Department of Mathematics \\ Utkal University \\ Vani Bihar \\ Bhubaneswar \\ Orissa, India \\ and
}

\section{B.K. PATEL}

Department of Mathematics

Khallikote College

Berhampur

Orissa

India, Pin Code - 760001

(Received January 21, 1986)

ABSTRACT. By introducing a sublinear functional involving infinite matrices, we establish its connection with ergodicity and measure preserving transformation. Further, we characterize the existence of a finite invariant measure by means of a condition involving the above sublinear functional.

KEY WORDS AND PHRASES. Sublinear functional, Banach limit, conservative, regular, translative, null invariant, measure preserving, ergodic.

1980 AMS SUBJECT CLASSIFICATION CODES. 58F11

1. INTRODUCTION AND DEFINITIONS.

$$
\text { Let } \ell_{\infty} \text { be the set of all real bounded sequence }\left\{x_{n}\right\} \text {, normed by }|| x||=\sup _{n \geq 0}\left|x_{n}\right|
$$

Linear functional $\phi$ on $\ell_{\infty}$ are called Banach limit [1] satisfying the conditions,

i) $\phi\left(x_{n}\right) \geq 0$, if $x_{n} \geq 0, n=0,1,2 \ldots$.

ii) $\phi\left(\mathrm{x}_{\mathrm{n}+1}\right)=\phi\left(\mathrm{x}_{\mathrm{n}}\right)$

iii) $\frac{\lim }{n \rightarrow \infty} x_{n} \leq \phi\left(x_{n}\right) \leq \prod_{n \rightarrow \infty} x_{n}$.

If there is a number for all Banach limits $\phi$, the sequence $x=\left\{x_{n}\right\}$ is called almost convergent and we write; $F-\lim _{n \rightarrow \infty} x_{n}=s$. It is shown by Lorentz [2] that a sequence $\left\{x_{n}\right\}$ is almost convergent with F-limit $s$, if and only if

$$
\lim _{n \rightarrow \infty} \frac{1}{n} \sum_{k=i}^{i+n-1} x_{k}=s .
$$

uniformly in $i$. 
Let, $A=\left(a_{n, k}(i)\right.$ be a sequence of real or complex matrices for each $i=0,1,2 \ldots$ such that $a_{n, k}(i)=0$, if any $n, k, i$, is a negative integer. The sequence $\left\{x_{n}\right\}$ is called A summable to $s$ if

$$
\lim _{n \rightarrow \infty} \sum_{k=0}^{\infty} a_{n, k}(i) x_{k}=s
$$

uniformly in $i$ and in this case we write:

$$
A-\lim _{n \rightarrow \infty} x_{n}=s \text {, or } x_{n} \rightarrow s(A) \text {. }
$$

In the case $a_{n, k}(i)=1 / n+1 \quad(i \leq k \leq i+n)$ and 0 otherwise, (A) reduces to the method (F). If $A=A=a_{n, k}$, then we obtain the usual summability method (A). It is significant to note that there does not exist any regular method (A) equivalent to $\operatorname{method}(F)$ (See Lorentz [2]. Theorem 11 and 12). In the case $a_{n, k}(i)=\frac{1}{n+1}{ }_{r=i}^{n+i} a_{r, k}$, then (A) reduces to the almost summability method introduced by King [3].

The method (A) is called conservative, if $x \rightarrow s=x \rightarrow s^{1}(A)$, regular, if $s=\dot{s}^{1}$. The following characterization of regular matrices is due to Stieglitz [4]. The method $(A)$ is called regular if and only if the following conditions hold:

$$
\sum_{k=0}^{\infty}\left|a_{n, k}(i)\right|<\infty \text {, for } a 11 n \text { and } i \geq 0 \text {, }
$$

and there exists an integer $m$ such that

$$
\begin{aligned}
& \sup _{i \geq 0, n \geq m} \stackrel{\infty}{\Sigma}=0_{n}\left|a_{n, k}^{(i)}\right|<\infty \\
& \lim _{n \rightarrow \infty} \sum_{k=0}^{\infty} a_{n, k}(i)=1 \text {, uniform } 1 y \text { in } i \text {, } \\
& \lim _{n \rightarrow \infty} \underset{n, k}{a(i)}=0 \text {, for fixed } k \text {, uniformly in } i \text {. }
\end{aligned}
$$

We write

$$
|| A||=\sup _{i \geq 0, n \geq 0} k_{=0}^{\infty}\left|a_{n, k}^{(i)}\right|
$$

The matrix $A$ is called translative, if

$$
\lim _{n \rightarrow \infty} \sum_{k=0}^{\infty}\left|d_{n, k}^{(i)}\right|=0
$$

uniformly in $i$, where

$$
d_{n, k}^{(i)}=\left(a_{n, k-1}^{(i)}-a_{n, k}^{(i)}\right)
$$

The matrix $A$ is called positive, if

$$
a_{n, k}^{(i)} \geq 0, \forall n, k, i
$$

For real $\lambda$ we write,

$$
\lambda^{+}=\max (\lambda, 0), \quad \lambda^{-}=\max (-\lambda, 0) .
$$

The matrix $A$ is called almost positive, if

$$
\lim _{n \rightarrow \infty} \sum_{k=0}^{\infty} a_{n, k}^{-(i)}=0 \text {, uniform1y in } i \text {. }
$$


Let $(\mathrm{x}, F, \mathrm{~m})$ be a finite measure space and let, $\mathrm{T} ; \mathrm{X} \rightarrow \mathrm{X}$ be a measurable transformation. (This is assumed throughout). The measure $m$ is called null invariant, if $m(A)=0<->m\left(T^{-1} A\right)=0, A \leftarrow F$. It is conservative, if $A \cap T^{-n} A=\phi \Rightarrow m(A)=0$, for all $\mathrm{n}$, and $A \in F$. A measure $\mu$ is called equivalent to measure $\mathrm{m}$, if $\mathrm{m}(\mathrm{A}) \Leftrightarrow \mu(\mathrm{A})=0$, for $\mathrm{A} \in \mathrm{F}$. The transformation $\mathrm{T}$ is called measure preserving or invariant, if $\mathrm{m}(\mathrm{A})=\mathrm{m}\left(\mathrm{T}^{-1} \mathrm{~A}\right), A \in F$. It is called ergodic if, $\mathrm{T}^{-1} \mathrm{~A}=\mathrm{A}=>\mathrm{m}(\mathrm{A})=0$ or $m(X / A)=0$. The set $A \in F$ is called invariant, if $A=T^{-1} A$. It is called wandering, if $A, T^{-1} A, T^{-2} A \ldots A \in F$, are mutually disjoint. It is called weakly wandering, if there is an increasing sequence of positive integers $\left\{r_{k}, k=1,2,3 \ldots\right\}$ such that $A, T^{-r_{1 A}}, T^{-r} 2 A \ldots$ are mutually disjoint. A measure $q$ is called $m-$ continuous if, for $\varepsilon>0$ there exists a $\delta>0$ such that $m(A)<\delta \Rightarrow q(A)<\varepsilon$. A sequence of measures $\left\{q_{n}\right\}$ is called uniformly m continuous if for each $\varepsilon>0$, there exists a $\delta>0$ such that $m(A)<\delta \Rightarrow q_{n}(A)<\varepsilon$ for all $n$.

Write:

$$
t(x)=\overline{1 i m}_{n \rightarrow \infty} \sup _{i} \frac{1}{n} \underset{k=i}{i+n-1} \quad x_{k}, \quad x_{k} \epsilon \ell_{\infty}
$$

Let $\left\{1_{\infty}, t\right\}$ denote the set of linear functionals $\phi$, such that $\phi(x) \leq t(x)$. It is known (see Sucheston [5] Das and Misra [6]) that $\left\{1_{\infty}, t\right\}$ is the set of all Banach limits on $l_{\infty}$ and $\phi \in\left\{1_{\infty}, t\right\}$ is unique if and only if $\phi(x)=-\phi(-x)$ and this happens when

$$
\frac{1}{n} \sum_{k=i}^{i+n-1} \quad x_{k} \rightarrow a \text { 1imit }
$$

as $n \rightarrow \infty$, uniformly in $i$. Lorentz [2] calls all such sequences as almost convergent sequences. Let $A$ be real and such that ||$A||<\infty$. Then we define, $R: 1_{\infty} \rightarrow 1_{\infty}$ by

$$
R(x)=\overline{\lim }_{n \rightarrow \infty} \sup _{i} \sum_{k=0}^{\infty} a_{n, k}^{(i)} x_{k} .
$$

Since, for all $x \in 1_{\infty}$

$$
|R(x)| \leq\|x\||| A||<\infty \text {, }
$$

$R$ is finite valued. It is easy to see that it is a sublinear functional on $l_{\infty}$. By Hahn-Banach theorem there exists a linear functional $\phi$ on $1_{\infty}$ such that

$$
-R(-x) \leq \phi(x) \leq R(x), \quad x \in 1_{\infty}
$$

Let $\left\{1_{\infty}, R\right\}$ be the set of all linear functional $\phi$ satisfying (1.13). It is easily seen that $\phi$ is unique if and only if

$$
-R(-x)=R(x)
$$

and this happens if and only if

$$
\stackrel{\sum}{\sum}=0_{n} a_{n, k}^{(i)} x_{k} \rightarrow a \text { limit }
$$

as $n \rightarrow \infty$, uniformly in $i$.

We now state a lemma.

LEMMA 1. Let $x \in 1_{\infty}$, then

(a) 1 im $x_{n} \leq R(x) \leq \overline{\operatorname{Iim}} x_{n}$ 
if and only if $A$ is real, regular and almost positive.

(b) $-t(-x) \leq-R(-x) \leq R(x) \leq t(x)$

if and only if $A$ is regular, almost positive and translative.

(c) If $x$ is almost convergent to $s$, then

$$
\lim _{n \rightarrow \infty} \sum_{k=0}^{\infty} a_{n, k}^{(i)} x_{k}=s \text {, uniform1y in } i
$$

\section{ERGODICITY.}

In this section, we establish that the ergodicity and invariance can be established in terms of summability of a particular sequence and thus generalizes a result of (Sucheston [5], Theorem 3) involving almost convergence.

We now examine the following conditions:

(II) $\quad \lim _{n \rightarrow \infty} \sum_{k=0}^{\infty} a_{n, k}^{(i)} m\left(T^{-n} B\right.$ ก C $)=m(B) m(C)$

uuiformly in i, $\mathrm{V} B, C \in F$.

(III) $\mathrm{T}$ is ergodic and measure preserving.

THEOREM 1. Let (X, $F, m)$ be a finite measure space and let ||$A||<\infty$. Then

(a) (II) $\Rightarrow$ (I)

(b) (i) (I) $\Rightarrow \mathrm{T}$ is ergodic

(ii) If $A$ is translative. Then

(I) $\Rightarrow$ (III)

(c) If $A$ is regular, almost positive, and translative, then

$$
\text { (I) } \Leftrightarrow \text { (II) } \Leftrightarrow \text { (III) }
$$

We need the following lemma for the proof of the theorem.

LEMMA 2. Let $\|A\|<\infty, \phi \in\left\{1_{\infty}, R\right\}, s: 1_{\infty} \rightarrow 1_{\infty}$ be the shift operator i.e.

$$
s\left(x_{n}\right)=x_{n+1}, \quad s^{2}\left(x_{n}\right)=x_{n+2} \text {. }
$$

Then

(a) $|\phi(s x)-\phi(x)| \leq|| x|| \frac{\lim _{n \rightarrow \infty}}{\sup _{i}} \sum_{k=0}^{\infty}\left|d_{n, k}^{(i)}\right|$

where $d_{n, k}^{(i)}$ is defined by (1.8).

Let $A$ be translative, then for $x \in 1_{\infty}$.

(b) (i) $\mathrm{R}(\mathrm{S} X-\mathrm{x})=\mathrm{R}(\mathrm{x}-\mathrm{S} X)=0$

(ii) $\phi(\mathrm{Sx})=\phi(\mathrm{x})$

(c) $\quad \mathrm{R}(\mathrm{Sx})=\mathrm{R}(\mathrm{x})$

Let, further

$$
\lim _{n \rightarrow \infty} a_{n, k}^{(i)}=0 \text {, fixed } k \text {, uniformly in } i
$$

Then

(d) $R\left(\sum_{j=0}^{p} s^{r j} x\right)=p \cdot R(x)$

Where $r_{0}=1, r_{1}, r_{2} \ldots r_{p}$ is a sequence of fixed positive integers.

PROOF: Since

$$
R(S x-x)=\varlimsup_{n \rightarrow \infty} \sup _{i} \sum_{k=0}^{\infty} a_{n, k}^{(i)}\left(S x_{k}-x_{k}\right)
$$




$$
=\lim _{n \rightarrow \infty} \sup _{i} \stackrel{\sum}{=}=0_{n, k} d_{n, k}^{(i)} x_{k} \text {. }
$$

It follows that

$$
|R(S x-x)| \leq|| x|| \lim _{n \rightarrow \infty} \sup _{i} \sum_{k=0}^{\infty}\left|d_{n, k}^{(i)}\right| .
$$

Now as $\phi$ is linear, we obtain

$$
\phi(s x)-\phi(x)=\phi(s x-x) \leq R(s x-x) .
$$

Changing the role of $s x$ and $x$ in (2.2) and (2.3) we obtain (a). When $A$ is translative (b) (i), (ii) follows from (2.2), and changing the role of $s x$ and $x$ in (2.2) (b) (ii) follows from (a). Since, $R$ is sublinear,

$$
R(S x)=R(S x-x+x) \leq R(S x-x)+R(x)=R(x)
$$

by $b$ (i). Changing the role of $S x$ and $x$, we obtain $R(x) \leq R(S x)$. So (c) follows. Lastly $R\left(S^{r}{ }^{1} x+S^{r}{ }^{2} x\right)=R\left(S^{r}{ }^{1} x-x+S^{r}{ }^{2} x-x+2 x\right) \leq R\left(S^{r}{ }^{1} x-x\right)+R\left(S^{r}{ }^{2}-x\right)+2 R(x)$. i.e.

$$
R\left(S^{r}{ }^{1} x+S^{r} x\right)-2 R(x) \leq R\left(S^{r} 1 x-x\right)+R\left(S^{r}{ }^{2} x-x\right)
$$

But,

$$
\begin{aligned}
& R\left(S^{r} 1_{x}-x\right)=\lim _{n \rightarrow \infty} \sup _{i} \sum_{k=0}^{\infty} a_{n, k}^{(i)}\left(x_{k+r}-x_{k}\right) \\
& =\lim _{n \rightarrow \infty} \sup _{i} \sum_{k=0}^{\infty}\left(\begin{array}{l}
(i) \\
n, k-r_{1}
\end{array}-a_{n, k}^{(i)}\right) x_{k} \\
& =\lim _{n \rightarrow \infty} \sup _{i}\left[\sum_{k=r_{1}}^{\infty}\left(a_{n, k-r_{1}}^{(i)}-a_{n, k}^{(i)}\right) x_{k}-{ }_{k=0}^{n-1} \underset{n, k}{a_{n}} \stackrel{(i)}{x_{k}}\right] \\
& =\lim _{n \rightarrow \infty} \sup _{i}\left[\sum_{k=r_{1}}^{\infty}\left(a_{n, k-r_{1}}^{(i)}-a_{n, k}^{(i)} x_{k}\right] \quad\right. \text { by (2.1) } \\
& =1 \overline{i m} \sup _{n \rightarrow \infty} \sum_{k=r}^{\infty} x_{k}{ }_{j=0}^{r_{1}-1}\left(a_{n, k-j-1}^{(i)}-a_{n, k-j}^{(i)}\right) \\
& \leq|| x|| \quad \sum_{j=0}^{r_{1}^{-1}} \underset{n \rightarrow \infty}{1-i m} \sup _{i} \sum_{k=r_{1}}^{\infty} \mid d_{n, k-j}(i) \\
& =0 \quad(\because A \text { is translative })
\end{aligned}
$$

Similarly

$$
R\left(S^{r} x-x\right) \leq 0
$$

Hence,

Again, since

$$
\mathrm{R}\left(\mathrm{s}^{\mathrm{r}}{ }^{1} \mathrm{x}+\mathrm{s}^{\mathrm{r}}{ }^{2} \mathrm{x}\right) \leq 2 \mathrm{R}(\mathrm{x}), \quad \mathrm{x} \in 1_{\infty} .
$$

$$
\begin{aligned}
& 2 R(x)=R\left(2 x-S^{r} 1 x-S^{r} 2 x+s^{r} 1 x+s^{r}{ }^{r} x\right) \\
& \leq R\left(x-S^{r}{ }^{1} x\right)+R\left(S-S^{r}{ }^{r} x\right)+R\left(S^{r}{ }^{1} x+S^{r}{ }^{r}\right) .
\end{aligned}
$$

Proceeding as above, we have

$$
2 R(x) \leq R\left(S^{r} 1_{x}+S^{r}{ }^{2} x\right), \quad x \in 1_{\infty} .
$$

Hence,

$$
R\left(S^{r} 1_{x}+S^{r}{ }^{2} x\right)=2 R(x), x \in 1_{\infty} .
$$

(d) follows by repeated application of (2.4). 
PROOF OF THEOREM 1.

(a) Let (II) hold. Then

$$
-R\left[-m\left(T^{-n} B \cap C\right)\right]=R\left[m\left(T^{-n} B \cap C\right)\right]
$$

Since

It follows that

$$
-R(x) \leq Q(x) \leq R(x), \quad x \in 1_{\infty}
$$

$$
\phi\left[m\left(T^{-n} B \cap C\right)\right]=m(B) \cdot m(C), n=0,1,2 \ldots .
$$

This proves (II) $\Rightarrow$ (I).

(b) Take, $\mathrm{T}^{-1} \mathrm{~B}=\mathrm{B}, \quad \mathrm{C}=\mathrm{x} / \mathrm{B}=\mathrm{B}^{-1}$ in (I).

Hence it follows that

$$
0=\phi(0)=\mathrm{m}(B) \cdot \mathrm{m}\left(\mathrm{B}^{1}\right)
$$

either $m(B)=0$ or $m\left(B^{l}\right)=0$.

i.e. $T$ is ergodic.

Writing, $C=x$ in (I), we obtain

$$
\phi\left[m\left(T^{-n} B\right)\right]=m(B) \cdot m(X)
$$

Replacing $B$ by $\mathrm{T}^{-1} \mathrm{~B}$ in (2.6), we obtain

$$
\phi\left[m\left(T^{-n-1} B\right)\right]=m\left(T^{-1} B\right) \cdot m(X)
$$

If further, $A$ is translative, by Lemma 2 (b)

$$
\phi\left[\mathrm{m}\left(\mathrm{T}^{-\mathrm{n}-1} B\right)\right]=\phi\left[\mathrm{m}\left(\mathrm{T}^{-1} B\right)\right] .
$$

Again, since $0<m(X)<\infty$, it follows from (2.6) and (2.7) that

$$
m\left(T^{-1} B\right)=m(B)
$$

Hence, (I) $\Rightarrow$ (III).

(c) In veiw of (a) and (b), it is enough to show that (III) $\Rightarrow$ (II). Take any fixed $B \in F$ such that $m(B)>0$. Define, for $\phi \in\left\{1_{\infty}, R\right\}$ and $C \in F$

$$
\begin{aligned}
& q_{n}(c)=\frac{m\left(T^{\left.-n_{B \cap C}\right)}\right.}{m(B)}, n=0,1,2 \ldots \\
& q(c)=\phi\left(q_{n}(c)\right)
\end{aligned}
$$

We now show that $q$ is an invariant measure and $m=q$.

Since, $A$ is almost positive

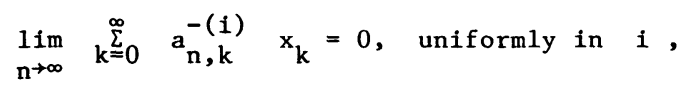

for $x_{k} \in 1_{\infty}$.

Write

$$
R^{+}(x)=\lim _{n \rightarrow \infty} \sup _{i} \sum_{k=0}^{\infty} a_{n, k}^{+(i)} x_{k}
$$

So, by (2.9)

$$
\begin{gathered}
R(x)=R^{+}(x) \\
\text { Since, } x \geq 0 \Rightarrow R^{+}(x) \geq 0 \\
x \geq 0 \quad \Rightarrow R(x) \geq 0 .
\end{gathered}
$$

Again, since $m$ is a measure, $q_{n}(c) \geq 0$. So it follows from (2.10) that $R\left(q_{n}(c)\right) \geq 0$. 


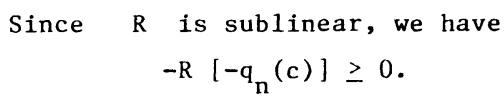

Now, it follows from (2.5) that $q(c) \geq 0, C \in F$. Let $B_{i} \in F$ be a countable sequence of disjoint sets. Then

$$
\begin{aligned}
q\left(\stackrel{\cup}{i=1}_{i}^{\infty} B_{i}\right) & =\phi\left[q_{n}\left(\stackrel{\cup}{i}=1_{i}^{\infty} B_{i}\right)\right] \\
& =\phi\left[\sum_{i=1}^{\infty} q_{n}\left(B_{i}\right)\right] \quad(. m \text { is a measure }) \\
& =\sum_{i=1}^{\infty} \phi\left[q_{n}\left(B_{i}\right)\right] \quad(\phi \text { is continuous 1inear functiona } 1)
\end{aligned}
$$

So, $q$ is countably additive and hence it is a measure.

Next,

$$
\begin{aligned}
\mathrm{q}\left(\mathrm{T}^{-1} \mathrm{C}\right) & =\phi\left[\frac{\mathrm{m}\left(\mathrm{T}^{-\mathrm{n}} \mathrm{B \cap T} \mathrm{T}^{-1} \mathrm{C}\right)}{\mathrm{m}(\bar{B})}\right] \\
& =\phi\left[\frac{\mathrm{m}\left(\mathrm{T}^{-\mathrm{n}+1} \mathrm{BnC}\right)}{\mathrm{m}(\mathrm{B})}\right] \quad(\cdot \mathrm{T} \text { is a measure preserving }) \\
& =\phi\left[\mathrm{q}_{\mathrm{n}-1}(\mathrm{C})\right]
\end{aligned}
$$

Since $\phi$ is shift invariant by Lemma 2 (b),

$$
\begin{aligned}
& =\phi\left[q_{n}(C)\right] \\
& =q(C), \quad C \in F .
\end{aligned}
$$

This proves that $\mathrm{q}$ is an invariant measure. Sice $\mathrm{T}$ is ergodic, the invariant sets are of measure 0 or 1 . Since $m$ and $q$ are invariant measures, an invariant measure is determined by the value it takes on invariant sets (See Sucheston [8], Theroem 2), it follows that $\mathrm{q}=\mathrm{m}$.

Now, we have

$$
q(C)=m(C)=\phi\left[\frac{m\left(T^{-n} B \cap C\right.}{m(B)}-\right] \text { i.e. } \phi\left[m\left(T^{-n} B \cap C\right)\right]=m(B) \cdot m(C)
$$

Hence, $\phi$ is unique on $\left\{\mathrm{T}^{-\mathrm{n}} \mathrm{B} \cap \mathrm{C}\right\}, \mathrm{n}=0,1,2 \ldots$. But, $\phi \in\left\{1_{\infty}, \mathrm{R}\right\}$ has unique value $\lambda$ if and only if

$$
R(x)=-R(-x)=\lambda \text {. }
$$

Hence, it follows that

$$
R\left[m\left(T^{-n} B \cap C\right)\right]=-R\left[-m\left(T^{-n} B \cap C\right)\right]=m(B) \cdot m(C) .
$$

i.e. (II) holds and hence proves (c) completely.

3. EQUIVALENT MEASURES.

Many necessary and sufficient conditions have been determined for the existence of equivalent invariant measures (see Sucheston [7], [8], Mrs. Dowker [9], Ca1deron [10], and Hajian and Kakutani [11]). In the pointwise ergodic theorem of Birkhoff [12], it was necessary to take invariant measure, but Halmos [13] has shown that even if a measure is null invariant and conservative, an equivalent measure need not exist.

Sucheston [7], [8] has used Banach limit technique to prove the existence of invariant measures. We now generalize some of the theorems of Sucheston [5] involving almost convergence and some results of Mrs. Dowker on $(C, 1)$ convergence and establish the existence of invariant measure by using linear functional $\phi \in\left\{1_{\infty}, R\right\}$.

We now prove

THEOREM 2. Let $A$ be a real matrix such that ||$A||<\infty$ and let $A$ be almost positive and translative. Let $(x, F, m)$ be a finite measure space and $T$ be a measurable 
transformation. Then, the following condition are equivalent.

(I) There exists an equivalent finite invariant measure.

(II) For some $\phi \in\left\{1_{\infty}, R\right\}$ and a11 B $\in F$

$$
\mathrm{m}(\mathrm{B})>0 \Rightarrow \phi\left[\mathrm { m } \left(\mathrm{T}^{\left.\left.-\mathrm{n}_{\mathrm{B}}\right)\right]>0}\right.\right.
$$

$$
\mathrm{m}(\mathrm{B})>0 \Rightarrow \mathrm{R}\left[\mathrm{m}\left(\mathrm{T}^{-\mathrm{n}} \mathrm{B}\right)\right]>0
$$

PROOF. (I) $\Rightarrow$ (II). Suppose that $p$ is an invariant measure which is equivalent to m. Suppose that (II) fails to hold. Then there exists a $B \in F$ such that $m(B)>0$ and

$$
\phi\left[m\left(T^{-n} B\right)\right]=0 .
$$

But, since $-R(-x) \leq \phi(x) \leq R(x), x \in 1_{\infty}$ and by Lemma 1

$$
\lim _{n \rightarrow \infty} x_{n} \leq-R(-x) \leq R(x) \leq \lim _{n \rightarrow \infty} x_{n} .
$$

it. follows that for all $B \in F$

$$
0=\phi\left[m\left(T^{-n} B\right)\right] \geq \frac{1 i m}{n \rightarrow \infty} m\left(T^{-n} B\right) .
$$

But, since $\frac{1 i m}{n \rightarrow \infty} m\left(T^{-n} B\right) \supseteq 0$, it follows that

$$
\frac{1 i m}{n \rightarrow \infty} m\left(T^{-n} B\right)=0 .
$$

Hence, there exists a sub sequence $\left\{x_{k}\right\}$ such that

$$
\frac{1 i m}{k \rightarrow \infty} m\left(T^{-n k} B\right)=0 \text {. }
$$

Since $p$ is equivalent to $m$, we obtain

$$
\mathrm{p}(\mathrm{B})>0 \text { and } \lim _{\mathrm{k} \rightarrow \infty} \mathrm{p}\left(\mathrm{T}^{-\mathrm{nk}} \mathrm{B}\right)=0 \text {. }
$$

Since $p$ is invariant, we have

$$
\mathrm{p}\left(\mathrm{T}^{-\mathrm{nk}} \mathrm{B}\right)=\mathrm{p}(\mathrm{B}) \text {. }
$$

Hence $p(B)=0$. This is a contradiction and this proves the fact that (I) $\Rightarrow$ (II).

$($ II $)=>$ (III) . Let II hold. Since, $\left.\phi\left[m^{-T^{-n}}\right)\right] \leq R\left[m\left(T^{-n_{B}}\right)\right]$ it follows that

$$
\phi\left[\mathrm{m}\left(\mathrm{T}^{-\mathrm{n}} \mathrm{B}\right)\right]>0 \Rightarrow \mathrm{R}\left[\mathrm{m}\left(\mathrm{T}^{-\mathrm{n}} \mathrm{B}\right)\right]>0 \text {. }
$$

(III) $\Rightarrow$ (I) . Suppose (III) holds and (I) fails. Since Condition (I) is equivalent to non-existence of weakly wandering set (See Sucheston [7], Theorem 6) it follows that there exists positive integers $r_{0}=1, r_{1}, r_{2} \ldots$ and a set $B \in F$ with $m(B)>0$ such that

$$
\mathrm{B}, \mathrm{T}^{-\mathrm{r}_{1}} \mathrm{~B}, \mathrm{~T}^{-\mathrm{r}_{2}} \mathrm{~B}, \ldots, \mathrm{T}^{-\mathrm{r}_{\mathrm{k}}} \mathrm{B} \ldots
$$

are mutually disjoint. Since,

$$
\begin{aligned}
& \lim _{n \rightarrow \infty} \sum_{k=0}^{\infty} a_{n, k}(i)=1 \text {, uniformly in } i \text {, it follows that } \\
& R\left[m\left(T^{-k} X\right)\right]=R[m(X)]=m(X) R(1)=m(X) .
\end{aligned}
$$

Again

$$
m(X)=R\left[m\left(T^{-n} X\right)\right] \geq R\left[m\left(\bigcup_{j=0}^{S} T^{-r j} B\right)\right]
$$




$$
\begin{aligned}
& m(X)=\underset{n \rightarrow \infty}{1 \overline{i m}} \sup _{i} \sum_{k=0}^{\infty} a_{n, k}^{(i)} m\left[\underset{j=0}{s} T^{-r j-k_{B}}\right] \\
& =\lim _{n \rightarrow \infty} \sup _{i} \sum_{k=0}^{\infty} a_{n, k}^{(i)} \quad j=0 \quad s^{r j} x_{k} .
\end{aligned}
$$

Where $X_{k}=m\left(T^{-k} B\right), S$ is a shift operator. Then by Lemma $2(d)$,

$$
\mathrm{m}(\mathrm{X}) \geq \mathrm{S} \cdot \mathrm{R}(\mathrm{X})
$$

Then it follows from (3.1) that

$$
\mathrm{m}(\mathrm{X}) \geq \mathrm{s} \cdot \mathrm{R} \quad \mathrm{m}\left(\mathrm{T}^{-\mathrm{k}} \mathrm{B}\right) \text {. }
$$

Since $\mathrm{m}\left(\mathrm{T}^{-\mathrm{k}} \mathrm{S}\right)>0$ by hypothesis and $\mathrm{s}$ is an arbitrary positive integer. This contradicts (3.2). This proves (III) $\Rightarrow$ (I).

In the next theorem we give yet another characterization of existence of invariant measure in terms of the sublinear functional $R(x)$.

THEOREM 3. Let $A$ satisfy the condition of Theorem 2 . Let $(x, F, m)$ be the finite measure space. Then there exists an invariant measrure equivalent to measure $m$ on $x$, if and only if,

(i) $\quad m$ is null-preserving.

(ii) $\mathrm{T}$ is conservative.

(iii) $\sum_{n=0}^{\infty} a_{n, k}(i) m\left(T^{\left.-k_{B}\right)}\right.$ converges uniformly in $i$, for every $B \in F$.

Again, whenever it has equivalent invariant measure, then the map $q: F \rightarrow R$

defined by $q(B)=R\left[m\left(T^{-n_{B}}\right)\right]$ is an invariant measure equivalent to $m$ and agrees with $m$ on invariant sets.

PROOF: NECESSITY .

Let us assume that $m$ admits an invariant equivalent measure $\mu$. Then $\mu$ is $\mathrm{m}$ continuous (See Halmos [9] p. 125).

Write for $\phi \in\left\{1_{\infty}, R\right\}$

$$
q(B)=\phi\left[m\left(T^{-n} B\right)\right]
$$

We want to show
(a) $\mathrm{q}$ is a measure
(b) $\mathrm{q}$ is a $\mathrm{m}$ continuous
(c) $q$ is invariant.

As in the proof of Theorem 1 , we can show that

$$
q(B) \geq 0 \text {, for all B } \in F \text {. }
$$

It is easy to show that

$$
B, C \in F, \quad B \subset C \Rightarrow q(B) \leq q(C) \text {. }
$$

Since $\phi$ is linear, it also follows that $q$ is finitely additive. Since $\mu$ is m-continuous, for given $\varepsilon>0, \delta>0$. Such that

$$
m\left(T^{-n} B\right)<\varepsilon \text { when } \mu(B)=\mu\left(T^{-n} B\right)<\delta \text {, and } m\left(T^{-n_{B}}<\varepsilon \Rightarrow q(B)<\varepsilon\right. \text {. }
$$

So $q$ is m-continuous. The countably additivity of $m$ and m-continuity of $q$ (See Halmos [9] p. 39).

Next,

$$
\begin{aligned}
q\left(T^{-1} B\right)-q(B) & \left.=\phi\left[m\left(T^{-n-1} B\right)\right]-\phi\left[m^{-n} T^{-n}\right)\right] \\
& =\phi\left[m\left(T^{-n-1} B\right)-m\left(T^{-n} B\right)\right](\phi \text { is 1inear }) .
\end{aligned}
$$


Since $\quad a_{n-1}(i)=0, \forall n$ and $i$

$$
\begin{aligned}
& \leq R\left[m\left(T^{-n-1} B\right)-m\left(T^{-n} B\right)\right] \\
& =\overline{1 i m}_{n} \sup _{i} \sum_{k=0}^{\infty} a_{n, k}^{(i)}\left[m\left(T^{-k-1} B\right)-m\left(T^{-k} B\right)\right] .
\end{aligned}
$$

$$
\begin{aligned}
& =\overline{\lim } \sup _{i} \sum_{k=0}^{\infty}\left[a_{n, k-1}(i)-a_{n, k}^{(i)}\right] m\left(T^{-k} B\right) \\
& \leq m(X) \lim _{n \rightarrow \infty} \sup _{i} \stackrel{\infty}{=}=0_{n=1}^{(i)} \mid .
\end{aligned}
$$

Since $A$ is translative,

Hence,

$$
\rightarrow 0 \text { as } n \rightarrow \infty \text {, uniformly in } i \text {. }
$$

$$
q\left(T^{-1} B\right) \leq q(B) \text {. }
$$

Changing the role of $T^{-1} B$ and $B$, we obtain

$$
q(B) \leq q\left(T^{-1} B\right) \text {. }
$$

Hence,

$$
q\left(T^{-1} B\right)=q(B)
$$

i.e. $q$ is invariant under $T$. Now if $\mathrm{T}^{-1} \mathrm{~B}=\mathrm{B}$. Then,

$$
\begin{aligned}
q(B) & =\phi\left[m\left(T^{-1} B\right)\right]=\phi[m(B)], \\
& =m(B) \cdot \phi(1)=m(B) .
\end{aligned}
$$

So $q=m$ on invariant sets. Hence (Sucheston [8], Theorem 2) $q=m$ on $F$. Thus

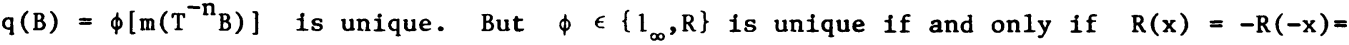
$q(B)$ and this happens if and only if

$$
\lim _{n \rightarrow \infty} \stackrel{\sum}{=}=0_{n, k}^{(i)} m\left(T^{-k} B\right)=q(B)
$$

uniformly in 1 .

$$
\text { Now since, } q\left(T^{-1} B\right)=q(B), B \in F \text { and } q=m \text { on } F \text {, we have } m\left(T^{-1} B\right)=m(B) \text {, }
$$
$B \in F$ so $\mathrm{m}(\mathrm{B})=0 \Rightarrow \mathrm{m}\left(\mathrm{T}^{-1} \mathrm{~B}\right)=0$

i.e. $m$ is null-preserving.

Again (See Sucheston [7], Theorem 6) existence of invariant measure is equivalent to non-existence of weakly wandering sets and non-existence of weakly wandering sets is the same as conservativeness of $T$.

SUFFICIENCY :

Let (i), (ii) and (iii) hold. Define

$$
q(B)=\lim _{n \rightarrow \infty} \stackrel{\infty}{\Sigma}=0_{n, k}^{(i)} m\left(T^{-k} B\right)
$$

Then it can be proved as before that $q$ ia an invariant measure. So only we have to prove $q$ is equivalent to $\mathrm{m}$. Since $\mathrm{T}$ is null preserving,

$$
m(B)=0 \Rightarrow m\left(T^{-1} B\right)=0 \text {. }
$$

Then

$$
q(B)=\lim _{n \rightarrow \infty} \sum_{k=0}^{\infty} a_{n, k}^{(i)} m\left(T^{\left.-k_{B}\right)}=0\right.
$$


Conversely, let $q(B)=0$.

Write;

$$
\begin{aligned}
& A^{*}=\stackrel{n}{n=1}_{i=n}^{\infty} T^{-i} B . \quad \text { Then } \\
& q\left(A^{*}\right)=q\left(\stackrel{n}{n=1}_{i=n}^{\infty} \stackrel{T}{ }^{-1} B\right) \\
& \leq q\left(\stackrel{\infty}{\stackrel{u}{N}_{1}} \mathrm{~T}^{-i} \mathrm{~B}\right) \\
& \leq \sum_{i=1}^{\infty} \mathrm{q}\left(\mathrm{T}^{-i} \mathrm{~B}\right) \quad(\mathrm{q} \text { is a measure }) \\
& =\sum_{i=n}^{\infty} q(B) \quad(q \text { is invariant) } \\
& =0
\end{aligned}
$$

Since, $q$ and $m$ agrees on invariant sets, we have $m\left(A^{*}\right)=0$. Since, $T$ is conservative by recurrence theorem $m\left(B / A^{*}\right)=0 \Rightarrow m(B)=0$.

Hence $q$ is equivalent to $\mathrm{m}$.

\section{REFERENCES}

1. BANACH, S., Theioris des Opirations Lineaires, New York, 1955.

2. LORENTZ, G. G., A Contribution to the Theory of Divergent Sequence, Acta. Math., 80 (1948), 187-190.

3. KING, J. P., Almost Summable Sequences, Proc. Amer. Math. Soc., (6) 17, 1966, 12191225.

4. STIEGLITZ, M., Eine Veral1gemeineinerungdes Begriffder Fastkonvergenz, Mathematica Japonicae, 18(1973), 53-70.

5. SUCHESTON, An Ergodic Application of Almost Convergence Sequence, Duke Math. Jour., 30(1963), 417-422.

6. DAS and MISRA, Sublinear Functiona1 and a Class of Conservative Matrices (Under Communication).

7. SUCHESTON, On Existence of Finite Invariant Measures, Math. Zeitschr, 86 (1964), 327336.

8. SUCHESTON, On the Ergodic Theorem for Positive Operators, $z$. Wahrscheinlichkeits Theorie, Und. Verw. Gebiete, B (1966), 215-218.

9. DOWKER, Y. N., On Measurable Transformations in Infinite Measure Space, Annals. of Math., (2) 62, (1955), 504-516.

10. CALDERON, A., Surles Measures Invarionts, C. R. Acad. Sci. Paris., 240 (1955), 19601962.

11. HAJIAN, A. and KAXUTANI, S., Weak1y Wandering Sets and Invartant Measures, Trans. Amer. Math. Soc., 110(1964), 136-151.

12. BIRKHOFF, G. D., Proof of the Ergodic Theorem, Proc. of National Aca. of Science, U.S.A., 17(1931), 656-660.

13. HALMOS, P. R., Lectures on Ergodic Theory, Che1sea, Pub1ishing, 1953.

14. HALMOS, P. R., Mesure Theory, NEW YARK VON NARSTAND, 1950.

15. DOWKER, Y. N., Finite and $\sigma$ Finite Invariant Measures, Annals. of Math., 54 (1951), 595-608. 


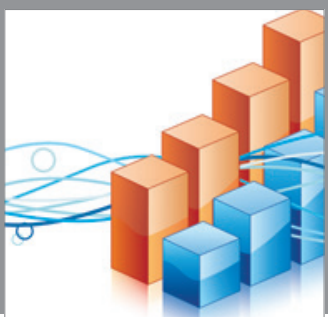

Advances in

Operations Research

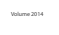

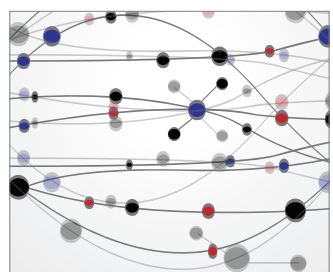

\section{The Scientific} World Journal
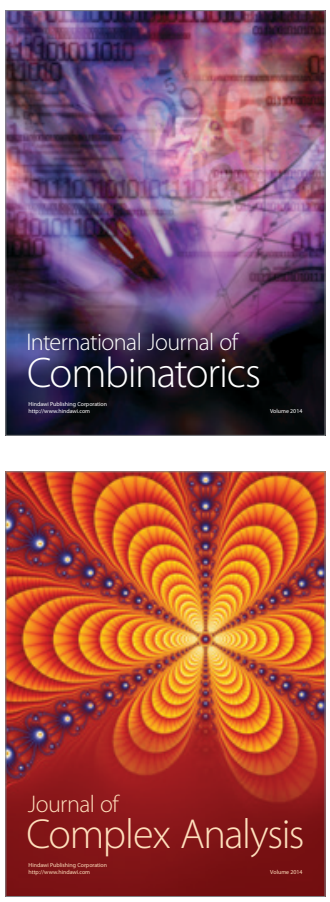

International Journal of

Mathematics and

Mathematical

Sciences
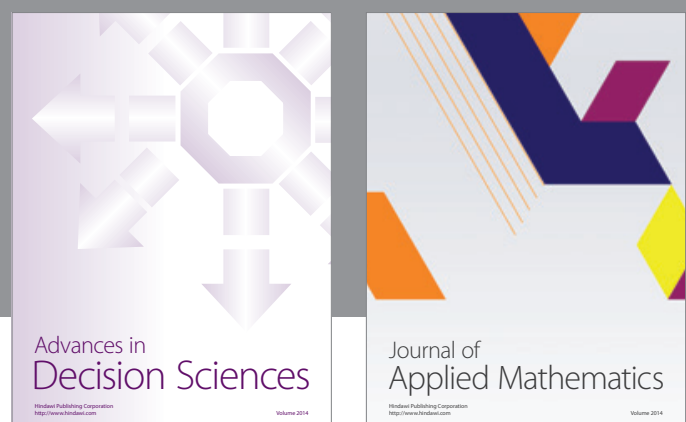

Journal of

Applied Mathematics
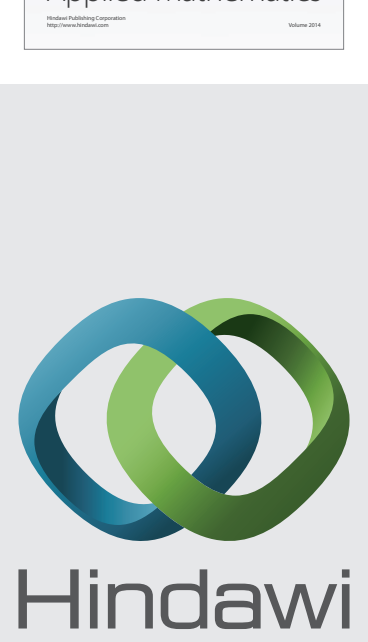

Submit your manuscripts at http://www.hindawi.com
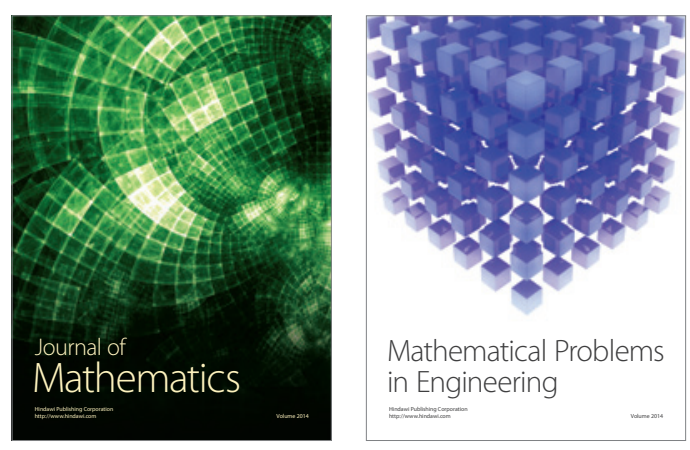

Mathematical Problems in Engineering
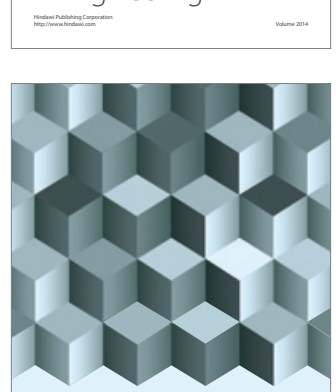

Journal of

Function Spaces
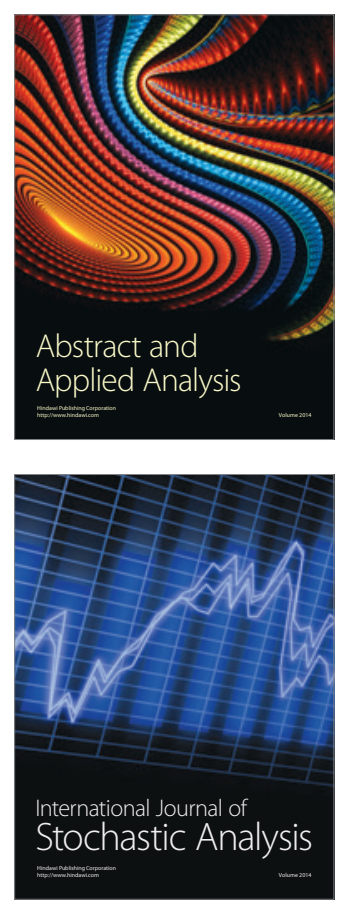

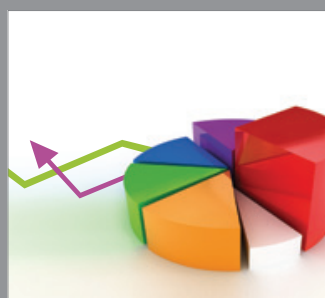

ournal of

Probability and Statistics

Promensencen
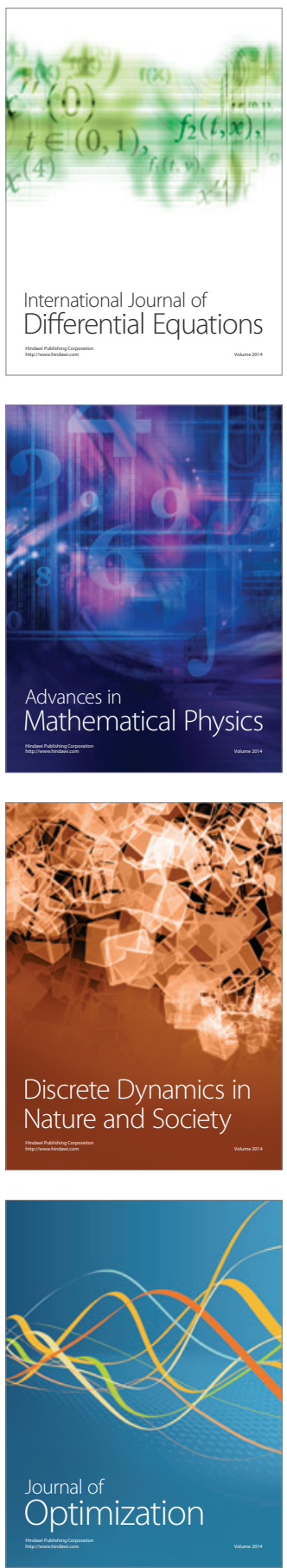